\title{
Computation of satellite clock-ephemeris augmentation parameters for dual-frequency multi-constellation satellite-based augmentation system
}

\author{
CHEN Jie, HUANG Zhigang, and LI Rui* \\ School of Electronic and Information Engineering, Beihang University, Beijing 100191, China
}

\begin{abstract}
Dual-frequency multi-constellation (DFMC) satellitebased augmentation system (SBAS) does not broadcast fast correction, which is important in reducing range error in L1-only SBAS. Meanwhile, the integrity bound of a satellite at low elevation is so loose that the service availability is decreased near the boundary of the service area. Therefore, the computation of satellite clockephemeris (SCE) augmentation parameters needs improvement. We propose a method introducing SCE prediction to eliminate most of the SCE error resulting from global navigation satellite system GNSS broadcast message. Compared with the signal-inspace (SIS) after applying augmentation parameters broadcast by the wide area augmentation system (WAAS), SIS accuracy after applying augmentation parameters computed by the proposed algorithm is improved and SIS integrity is ensured. With global positioning system (GPS) only, the availability of category-I (CAT-I) with a vertical alert level of $15 \mathrm{~m}$ in continental United States is about $90 \%$, while the availability in the other part of the WAAS service area is markedly improved. With measurements made by the stations from the crustal movement observation network of China, users in some part of China can obtain CAT-I (vertical alert limit is $15 \mathrm{~m}$ ) service with GPS and global navigation satellite system (GLONASS).
\end{abstract}

Keywords: dual-frequency multi-constellation (DFMC), satellitebased augmentation system (SBAS), orbit determination, integrity.

DOI: $10.21629 / J S E E .2018 .06 .01$

\section{Introduction}

In the next 10 years, global navigation satellite systems (GNSSs), including United States' global positioning system (GPS), Russian Federation's global navigation satellite system (GLONASS), Europe's Galileo system and China's BeiDou navigation satellite system (BDS), will provide two open signals for civil aviation. The first order effect of the ionosphere on ranging can be effectively eliminated with the combination of those dual-frequency

Manuscript received March 23, 2017.

*Corresponding author. signals. Compared with a single-frequency receiver, a dual-frequency receiver will thus obtain a more robust navigation service, especially in a region where the ionosphere is disturbed. Satellite-based augmentation system (SBAS) service providers plan to upgrade their systems to provide augmentation information for one or more GNSS constellations. The new system is called dual-frequency multi-constellation (DFMC) SBAS, whose interface control document (ICD) and definition document are currently being developed by the SBAS interoperability working group (IWG). The service providers of wide area augmentation system (WAAS), European geostationary navigation overlay system (EGNOS), and other SBASs are all participating in the SBAS IWG. According to the DFMC SBAS definition document v2.0 [1], GPS L1 C/A and L5 signals, Galileo E1 and E5a signals, and BDS B1C and B2a signals should be used by receivers operating in the DFMC SBAS mode, while GLONASS's signals for the DFMC SBAS mode are to be determined.

Satellite clock-ephemeris (SCE) errors, which are the differences between the true SCE values and SCE values computed using GNSS broadcast message, are estimated by the DFMC SBAS master station in real time. SCE augmentation parameters consist of SCE corrections, which are known as long-term satellite error corrections in the legacy L1-only SBAS, and the covariance matrix describing residual SCE after applying SCE corrections (hereafter called $\left.P_{\mathrm{SCE}}\right)$. The SCE augmentation parameters are computed based on the estimation of SCE errors.

DFMC SBAS users apply SCE corrections as follows:

$$
\left[\begin{array}{l}
x \\
y \\
z \\
t
\end{array}\right]_{\mathrm{SBAS}}=\left[\begin{array}{l}
x \\
y \\
z \\
t
\end{array}\right]_{B M}+\left[\begin{array}{c}
\delta x \\
\delta y \\
\delta z \\
\delta B
\end{array}\right]+\left[\begin{array}{c}
\delta \dot{x} \\
\delta \dot{y} \\
\delta \dot{z} \\
\delta \dot{B}
\end{array}\right]\left(t_{N}-t_{D}\right)
$$

where the vectors $\left[\begin{array}{llll}x & y & z & t\end{array}\right]_{\mathrm{SBAS}}^{\mathrm{T}}$ and $\left[\begin{array}{llll}x & y & z & t\end{array}\right]_{B M}^{\mathrm{T}}$ 
are SCE values computed using GNSS broadcast message at time $t_{N}$ with and without SCE corrections, respectively; $\delta x, \delta y, \delta z, \delta \dot{x}, \delta \dot{y}, \delta \dot{z}, \delta B$ and $\delta \dot{B}$ are SCE corrections, and $t_{D}$ is the reference time of SCE corrections. $\boldsymbol{P}_{\mathrm{SCE}}$ is used to compute the model variance of user range error (URE) resulting from residual SCE errors according to

$$
\sigma_{\mathrm{SCE}}^{2}=\boldsymbol{I}^{\mathrm{T}} \boldsymbol{P}_{\mathrm{SCE}} \boldsymbol{I}
$$

$I$ is the 4-D line of the sight vector, where the first three components are the unit vectors from the user to the satellite and the fourth component is 1 .

Enge et al. gave a complete description of the computation algorithm of the SCE augmentation parameters in the early WAAS [2]. The algorithm was designed for the GPS when the selective availability (SA) was used to intentionally degrade civil navigation performance. After the United States government announced that it had no intent to ever use SA again, the algorithms used in WAAS keep being upgraded, including measurement processing algorithms [3 - 5], ionospheric delay estimating algorithms [68], SCE determining and integrity monitoring algorithms $[9,10]$. A brief description of the two Kalman filters of the new method used in WAAS to determine SCE was given by Grewal [11]. The first filter focusing on the long-term SCE error iterates every $5 \mathrm{~min}$, while the second filter estimates the satellite clock error and iterates every second. However, no information about the state transition models was given in that paper. Thus, it is hard to reconstruct from the paper what the method actually is.

The details of the draft DFMC SBAS ICD have been described by Walter et al. [12]. We think it is noteworthy that a DFMC SBAS does not broadcast terms equivalent to fast corrections in an L1-only SBAS in order to augment as many as 92 satellites concurrently. This difference requires a major upgrade in the computation method of augmentation parameters because fast corrections improve range accuracy a lot. The Prototyping and Support to Standardization of SBAS L1/L5 Multi-Constellation Receiver (PROSBAS) project has been established [13]. The results of PROSBAS experimentation show that it may be risky to support category-I (CAT-I) operations with vertical alert limit (VAL) being $10 \mathrm{~m}$ in dual-frequency mode over the European civil aviation conference (ECAC) service area under EGNOS V3 reference conditions with the draft DFMC SBAS ICD [14]. There is only a few papers about the SCE augmentation algorithm for DFMC SBAS. One reason is that DFMC SBAS ICD has not been released to the public yet. Another reason may be that SBAS providers would like to keep core algorithms confidential.

Currently, BeiDou satellite-based augmentation system (BDSBAS) is under design and construction. It is essen- tial to establish an algorithm to compute SCE augmentation parameters with accuracy improved and integrity ensured. This paper is organized as follows: Section 2 decribes the over-bounded problem in WAAS. The method to compute SCE correction parameters and integrity information is presented in Section 3. Section 4 gives the evaluation results using 366-day measurements collected by WAAS stations, while Section 5 shows the preliminary results using 8-day measurements collected by crustal movement observation network of China (CMONOC) stations. Conclusions are drawn in Section 6.

\section{Problem statement}

Without considering the time differences between GNSSs, the weighted least-squares navigation solution is given by

$$
\widehat{\boldsymbol{X}}=\left(\boldsymbol{G}^{\mathrm{T}} \boldsymbol{W} \boldsymbol{G}\right)^{-1} \boldsymbol{G}^{\mathrm{T}} \boldsymbol{W} \boldsymbol{Y}
$$

where $\widehat{\boldsymbol{X}}$ is the weighted least-squares estimation of the error in the estimated user location about which the linearization has been made; $\boldsymbol{G}$ is the observation matrix, whose $i$ th row is $\left[-\cos \left(E l_{i}\right) \sin \left(A z_{i}\right) \quad-\cos \left(E l_{i}\right) \cos \left(A z_{i}\right)\right.$ $-\sin \left(E l_{i}\right)$ 1] with the elevation angel $E l_{i}$ and the azimuth angle $A z_{i}$ from the user to the $i$ th satellite; $\boldsymbol{Y}$ is an $N$-dimensional vector containing residual range errors, which are the combination of URE after applying SCE corrections, residual atmospheric delay, multipath delay and measurement noise; $N$ is the number of satellites used in the navigation solution; $\boldsymbol{W}=$ $\operatorname{diag}\left(1 / \sigma_{1}^{2}, 1 / \sigma_{2}^{2}, \ldots, 1 / \sigma_{N}^{2}\right)$ is a diagonal weight matrix; and $\sigma_{i}^{2}$ in the matrix $\boldsymbol{W}$ is the model variance of the residual range error of the $i$ th satellite.

The position error cannot be given in real time because the true position of a flying aircraft is usually unknown. Horizontal protection level (HPL) and vertical protection level (VPL), which describe the limits of horizontal position error (HPE) and vertical position error (VPE), are computed in real time to determine whether a certain operation is supported. HPL and VPL for a precision approach (PA) are given by

$$
\left\{\begin{array}{l}
H P L_{\mathrm{PA}}=K_{\mathrm{HPL}} \times d_{\mathrm{major}} \\
V P L_{\mathrm{PA}}=K_{\mathrm{VPL}} \times d_{U}
\end{array}\right.
$$

where $K_{\mathrm{HPL}}$ and $K_{\mathrm{VPL}}$ are 6.0 and $5.33 ; d_{\text {major }}$ and $d_{U}$ are computed as

$$
\left\{\begin{array}{l}
d_{\text {major }}=\sqrt{\frac{D_{1,1}^{2}+D_{2,2}^{2}}{2}+\sqrt{\left(\frac{D_{1,1}^{2}-D_{2,2}^{2}}{2}\right)^{2}+D_{1,2}^{2}}} \\
d_{U}=D_{3,3}
\end{array}\right.
$$

with $D_{i, j}$ being the $(i, j)$ th element of $\boldsymbol{D}=\left(\boldsymbol{G}^{\mathrm{T}} \boldsymbol{W} \boldsymbol{G}\right)^{-1}$ [15]. Thus, reducing $\sigma_{i}^{2}$ is the most efficient way to obtain 
lower protection levels without introducing more navigation satellites.

For a DFMC SBAS receiver, $\sigma_{i}^{2}$ is computed as

$$
\sigma_{i}^{2}=\sigma_{i, \mathrm{SCE} \_\mathrm{DF}}^{2}+\sigma_{i, \text { tropo }}^{2}+\sigma_{i, \text { air_DF }}^{2}
$$

where $\sigma_{\mathrm{SCE} \_\mathrm{DF}}^{2}$ is the model variance for the URE after applying SCE corrections provided by a DFMC SBAS; $\sigma_{\text {tropo }}^{2}$ is the model variance for the troposphere residual error; and $\sigma_{\text {air_DF }}^{2}$ is the model variance for the dualfrequency measurement noise, dual-frequency multipath error and ionospheric residual error after iono-free combination. Currently, 95\% pseudorange residual error of GPS satellites after applying WAAS corrections is from $0.670 \mathrm{~m}$ to $2.944 \mathrm{~m}$ as listed in [16]. 95\% URE after applying WAAS long-term corrections and fast corrections (hereafter called URE $E_{\text {FLT }}$ ) is about $0.7 \mathrm{~m}$. Considering that troposphere residual error, dual-frequency measurement noise, dual-frequency multipath error and ionospheric residual error after iono-free combination are all smaller than URE mentioned above in the nominal condition, we come to the conclusion that $\sigma_{\mathrm{SCE} \_\mathrm{DF}}^{2}$ is the dominant component of $\sigma_{i}^{2}$. To reduce the user protection level, the SBAS service provider should improve algorithms to decrease $\sigma_{\text {SCE_DF }}^{2}$.

The integrity relation between $\sigma_{\text {SCE_DF }}^{2}$ and URE after applying SCE corrections is given by

$$
\operatorname{Pr}\left[|\mathrm{URE}|<K_{\mathrm{HMI}} \sigma_{\mathrm{SCE} \_\mathrm{DF}}\right]<1-P_{\mathrm{HMI}}
$$

where $K_{\text {HMI }}$ is the quantile of the standard normal distribution with probability $1-P_{\mathrm{HMI}}$, which is the probability of there being hazardously misleading information of a signal-in-space (SIS). Obviously, an improvement on the accuracy of SCE corrections and $P_{\mathrm{SCE}}$ can reduce $\sigma_{\mathrm{SCE} \_D F}^{2}$ and thus lower the user protection level.

Fig. 1 shows the model standard deviation $\sigma_{\mathrm{FLT}}$ for $\mathrm{URE}_{\mathrm{FLT}}$ of GPS pseudorandom noise (PRN) 1 satellite on January 1,2016 . It is clear that $\sigma_{\mathrm{FLT}}$ can be very large while $\mathrm{URE}_{\mathrm{FLT}}$ is smaller than $2 \mathrm{~m}$ sometimes around 3.6 $\times 10^{4} \mathrm{~s}$ and $6.5 \times 10^{4} \mathrm{~s}$. That means at the beginning or the ending of the augmentation period, $\mathrm{URE}_{\mathrm{FLT}}$ is too over-bounded. We think a possible reason is that WAAS relies mainly on real-time measurement so that the strict requirement of the alert time of a precision approach can be met. Measurements are of poor quality when a satellite is at low elevation. This shortcoming enlarges the user protection level and thus decreases the availability near the boundary of the service area where many satellites in view are at the beginning or the ending of the augmentation period. In order to ensure that service availability is met all over the service area, the shortcoming should be overcome.

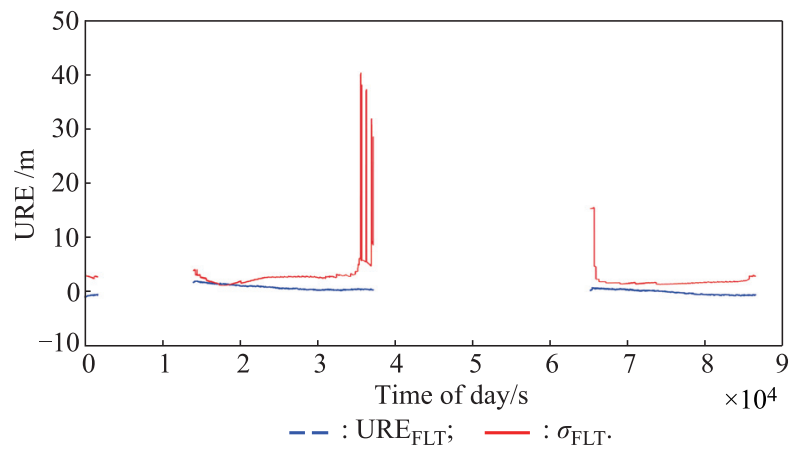

Fig. 1 URE $_{\text {FLT }}$ and $\sigma_{\text {FLT }}$ of GPS PRN 1 on January 1, 2016

A promising way to improve correction accuracy with the current configuration of WAAS stations is increasing the complexity of models used in the WAAS algorithm. However, the introduction of complex models increases the nonlinearity and computational burden, which is not suitable for an SBAS requiring high robustness and a strict alert time.

\section{SCE augmentation parameters computation}

It is necessary to analyze SCE error sources to find a way to improve SCE correction accuracy. A GNSS master station uses several-day-long measurements collected by stations around the world and highly accurate models to predict SCE values. Then, SCE parameters in GNSS broadcast message are computed based on these predictions. Therefore, the SCE error includes prediction error and parameter-fitting error during GNSS normal operations. The prediction error, which refers to the difference between SCE prediction and SCE true value, results from measurement error and prediction model inaccuracy. The parameter-fitting error, which refers to the difference between SCE prediction and SCE value computed with GNSS broadcast message, is caused by quantization error and model error of the broadcast message. Currently, the root mean square (RMS) value of a GPS satellite's URE is from $1.175 \mathrm{~m}$ to $2.006 \mathrm{~m}$, as listed in [17], while the SCE prediction is of centimeter-level accuracy as inferred from the accuracy of the International GNSS Service (IGS) ultra-rapid products given in [18]. The parameter-fitting error is thus the main source of SCE error.

We propose an algorithm that introduces SCE prediction to eliminate the parameter-fitting error and uses realtime measurements to reduce the prediction error, so that SCE augmentation parameters can be accurately computed in real time. For integrity reasons, an SBAS should be independent to the augmented GNSS. Therefore, SCE prediction should come from a trusted third-party source. The SCE prediction used in the proposed algorithm is based on the ultra-rapid ephemeris provided by the IGS. The 
parameter-fitting error is used as a priori knowledge of SCE error, which is defined as

$$
\boldsymbol{X}_{a p}=\boldsymbol{X}_{\text {pred }}-\boldsymbol{X}_{B M}
$$

where $\boldsymbol{X}_{a p}, \boldsymbol{X}_{\text {pred }}$ and $\boldsymbol{X}_{B M}$ are the a priori value of SCE error, SCE prediction value and SCE computed value using GNSS broadcast message, respectively. Because the basic idea of this algorithm is to introduce a priori knowledge of SCE error, it is hereafter called AK method.

\subsection{Computation of SCE error}

A minimum-variance unbiased estimator described in [19] is chosen to combine the a priori knowledge and real-time measurements:

$$
\left\{\begin{array}{l}
\boldsymbol{X}_{M V}=\boldsymbol{X}_{a p}+\boldsymbol{\Lambda} \boldsymbol{H}^{\mathrm{T}}\left(\boldsymbol{H} \boldsymbol{\Lambda} \boldsymbol{H}^{\mathrm{T}}+\boldsymbol{R}\right)^{-1}\left(\boldsymbol{Z}-\boldsymbol{H} \boldsymbol{X}_{a p}\right) \\
\boldsymbol{P}_{M V}=\boldsymbol{\Lambda}-\boldsymbol{\Lambda} \boldsymbol{H}^{\mathrm{T}}\left(\boldsymbol{H} \boldsymbol{\Lambda} \boldsymbol{H}^{\mathrm{T}}+\boldsymbol{R}\right)^{-1} \boldsymbol{H} \boldsymbol{\Lambda}
\end{array}\right.
$$

where $\boldsymbol{X}_{M V}$ is the estimation of SCE error; $\boldsymbol{Z}$ is a vector containing the residual range error based on the true station position and SCE value computed with the GNSS broadcast message; $\boldsymbol{P}_{M V}, \boldsymbol{\Lambda}$ and $\boldsymbol{R}$ are covariances of $\boldsymbol{X}_{M V}$, $\boldsymbol{X}_{a p}$ and $\boldsymbol{Z}$, respectively; and $\boldsymbol{H}$ is the observation matrix, whose $i$ th row is the 4-D line of sight vector, where the first three components are the unit vector from the station to the satellite and the fourth component is -1 . When a satellite is at a low elevation angle viewed from SBAS stations, measurements are of low quality and $\boldsymbol{R}$ is large, while $\boldsymbol{\Lambda}$ changes little with the elevation angle because GNSS stations are distributed worldwide. The weight of $\boldsymbol{Z}$ is thus smaller than that of $\boldsymbol{X}_{a p}$, which is helpful in terms of improving the estimation accuracy of a satellite at a low elevation angle. Therefore, the disadvantage of the WAASlike algorithm mentioned in the problem statement section is overcome.

There is a hidden problem in (9). When the monitoring stations change, a step response in $\boldsymbol{X}_{M V}$ may occur, which means there is a discontinuity in satellite movement. That is a violation of the laws of mechanics. To weaken the effect of the change in monitoring stations, we introduce a Kalman filter after the minimum-variance unbiased estimator. The key to Kalman filters is the state transition model. Given that a satellite's motion during a short period has an approximately constant speed and turn rate in its orbital plane, the planar constant turn (CT) model [20] is chosen to describe the transition in the ephemeris state. Although it is difficult to describe the transition in the satellite clock state as accurately as that in the ephemeris state, we choose the Singer model [21] to describe the satellite clock error on the basis that the clock drift rate can be treated as a time-correlated stochastic process.
The important elements of the Kalman filter are given below. The state equation is

$$
\boldsymbol{X}_{K}(k)=\boldsymbol{\Phi}_{K}(k) \boldsymbol{X}_{K}(k-1)+\boldsymbol{v}_{K}(k)
$$

where $\boldsymbol{X}_{K}(k), \boldsymbol{\Phi}_{K}(k)$ and $\boldsymbol{v}_{K}(k)$ denote the state vector, transition matrix and process noise vector at time $t_{k}$, respectively. The state vector $\boldsymbol{X}_{K}$ is given by

$$
\boldsymbol{X}_{K}=[x, \dot{x}, \ddot{x}, y, \dot{y}, \ddot{y}, z, \dot{z}, \ddot{z}, t, \dot{t}, \ddot{t}]^{\mathrm{T}}
$$

where $x, y, z$ and $t$ are satellite ephemeris and clock. The discrete-time state transition matrix $\boldsymbol{\Phi}_{K}$ is given by

$$
\boldsymbol{\Phi}_{K}=\left[\begin{array}{cc}
\boldsymbol{\Phi}_{\mathrm{CT}} & \mathbf{0} \\
\mathbf{0} & \boldsymbol{\Phi}_{\text {Singer }}
\end{array}\right]
$$

where $\boldsymbol{\Phi}_{\mathrm{CT}}$ and $\boldsymbol{\Phi}_{\text {Singer }}$ are the discrete-time state transition matrixes derived from the planar CT model and Singer model, respectively. The parameter $\alpha$ in the Singer model is set to $240 \mathrm{~s}$, which is equal to the time-out of SCE corrections for precision approach operations. The covariance of $\boldsymbol{v}_{K}$ is conservatively set to

$$
\boldsymbol{Q}_{v}=\left[\begin{array}{cc}
\boldsymbol{Q}_{\mathrm{CT}} & \mathbf{0} \\
\mathbf{0} & \boldsymbol{Q}_{\text {Singer }}
\end{array}\right]
$$

where $\boldsymbol{Q}_{\mathrm{CT}}$ is the covariance of the process noise with the power spectral density $\boldsymbol{S}_{\mathrm{CT}}$ in each Earth-centered inertial (ECI) axis in the planar CT model, and $\boldsymbol{Q}_{\text {Singer }}$ is the covariance of the process noise with the power spectral density $\boldsymbol{S}_{\text {Singer }}$ in the Singer model.

The observation equation is

$$
\boldsymbol{Z}_{K}(k)=\boldsymbol{H}_{K} \boldsymbol{X}_{K}(k)+\boldsymbol{\varepsilon}_{K}(k)
$$

where $\boldsymbol{Z}_{K}(k), \boldsymbol{H}_{K}(k)$ and $\boldsymbol{\varepsilon}_{K}(k)$ denote the observation vector, observation matrix and observation noise at time $t_{k}$, respectively. The observation vector $Z_{K}$ is calculated from $\boldsymbol{X}_{M V}$ as

$$
\boldsymbol{Z}_{K}=\boldsymbol{C}_{\mathrm{ECEF}}^{\mathrm{ECI}}\left(\boldsymbol{X}_{M V}+\boldsymbol{X}_{B M}\right)
$$

where $C_{\mathrm{ECEF}}^{\mathrm{ECI}}$ is the conversation matrix from the Earthcentered Earth-fixed (ECEF) frame to the Earth-centered inertial frame. The observation matrix $\boldsymbol{H}_{K}$ is timeinvariant:

$$
\boldsymbol{H}_{K}=\left[\begin{array}{l}
1,0,0,0,0,0,0,0,0,0,0,0 \\
0,0,0,1,0,0,0,0,0,0,0,0 \\
0,0,0,0,0,0,1,0,0,0,0,0 \\
0,0,0,0,0,0,0,0,0,1,0,0
\end{array}\right] .
$$

Because $\boldsymbol{X}_{M V}$ is treated as a random variable in (15), the observation error $\varepsilon_{K}$ comes from the uncertainty of $\boldsymbol{X}_{M V}$. With the independence between the planar CT model and Singer model, the covariances between the 
ephemeris and clock are zero. The covariance of $\varepsilon_{K}$ is thus conservatively set to

$$
\boldsymbol{R}_{\varepsilon}=C_{\mathrm{ECEF}}^{\mathrm{ECI}}\left[\begin{array}{cccc}
P_{1,1} & P_{1,2} & P_{1,3} & 0 \\
P_{2,1} & P_{2,2} & P_{2,3} & 0 \\
P_{3,1} & P_{3,2} & P_{3,3} & 0 \\
0 & 0 & 0 & P_{4,4}
\end{array}\right] \boldsymbol{C}_{\mathrm{ECEF}}^{\mathrm{ECI} \mathrm{T}}
$$

where $P_{i, j}$ is the $(i j)$ th element of $\boldsymbol{P}_{M V}$.

After the application of the minimum-variance unbiased estimator and the Kalman filter, the final SCE error estimation $\widehat{\boldsymbol{X}}_{e s t i}$ at time $t_{k}$ is

$$
\left\{\begin{array}{c}
\boldsymbol{X}_{e s t i}(k)=\left[\begin{array}{c}
\delta \widehat{x} \\
\delta \widehat{y} \\
\delta \widehat{z} \\
\delta \widehat{B}
\end{array}\right]=C_{\mathrm{ECEF}}^{\mathrm{ECI} T}\left[\begin{array}{c}
X_{1} \\
X_{4} \\
X_{7} \\
X_{10}
\end{array}\right]-\boldsymbol{X}_{B M}(k) \\
\boldsymbol{P}_{e s t i}(k)=C_{\mathrm{ECEF}}^{\mathrm{ECI}} . \\
{\left[\begin{array}{llll}
P_{1,1} & P_{1,4} & P_{1,7} & P_{1,10} \\
P_{4,1} & P_{4,4} & P_{4,7} & P_{4,10} \\
P_{7,1} & P_{7,4} & P_{7,7} & P_{7,10} \\
P_{10,1} & P_{10,4} & P_{10,7} & P_{10,10}
\end{array}\right] \boldsymbol{C}_{\mathrm{ECEF}}^{\mathrm{ECI}}}
\end{array}\right.
$$

where $X_{i}$ is the $i$ th element of $\boldsymbol{X}_{K}(k)$ and $P_{i, j}$ is the $(i, j)$ th element of $\boldsymbol{P}_{K}(k)$ in the Kalman filter.

\subsection{Computation of SCE correction parameters}

The SCE correction parameter vector $\boldsymbol{X}_{F}$ is computed every $120 \mathrm{~s}$ using a least-squares estimator with the SCE error estimations, which is computed every second, within the most recent $900 \mathrm{~s}$. For instance, $\delta x$ and $\delta \dot{x}$ in $\boldsymbol{X}_{F}$ are fitted as

$$
\left\{\begin{array}{l}
{\left[\begin{array}{c}
\delta x \\
\delta \dot{x}
\end{array}\right]_{F}=\left(\boldsymbol{H}_{F}^{\mathrm{T}} \boldsymbol{H}_{F}\right)^{-1} \boldsymbol{H}_{F}^{\mathrm{T}}\left[\begin{array}{c}
\delta \widehat{x}_{1} \\
\delta \widehat{x}_{2} \\
\vdots \\
\delta \widehat{x}_{m}
\end{array}\right]} \\
\boldsymbol{H}_{F}=\left[\begin{array}{cc}
1 & t_{1}-t_{0} \\
1 & t_{2}-t_{0} \\
\vdots & \vdots \\
1 & t_{m}-t_{0}
\end{array}\right]
\end{array}\right.
$$

where $\delta \widehat{x}_{i}$ is the first element of $\boldsymbol{X}_{\text {esti }}(i)$ at time $t_{i}, t_{0}$ is the reference time of SCE corrections, $m$ is the number of SCE error estimations used in (19). Then, $\boldsymbol{X}_{F}$ is discretized to SCE corrections parameters $\boldsymbol{X}_{F, b r d c}$ in Message Type 32 according to the DFMC SBAS ICD to be broadcast to users.

A solution to (9) is available as long as a measurement exists, which means a satellite can be augmented whenever it is tracked by any SBAS station. For integrity reasons, SCE error estimations used for SCE correction are computed when at least three stations are tracking the satellite.

\subsection{Computation of $\boldsymbol{P}_{\mathrm{SCE}}$}

There are four main reasons why it is not suitable to use $\boldsymbol{P}_{\text {esti }}$ as $\boldsymbol{P}_{\mathrm{SCE}}$. First, satellite clock is treated as an independent object to ephemeris in the Kalman filter, making $\boldsymbol{P}_{\text {esti }}$ not compatible with the real relation between ephemeris and clock. Second, the threats, such as the antenna bias and undetected measurement error, should be protected against. Third, SBAS receivers use $\boldsymbol{X}_{F, b r d c}$ instead of $\boldsymbol{X}_{\text {esti }}$ to correct SCE error, the difference between $\boldsymbol{X}_{F, b r d c}$ and $\boldsymbol{X}_{e s t i}$ thus must be included in $\boldsymbol{P}_{\mathrm{SCE}}$. Fourth, $\boldsymbol{P}_{\mathrm{SCE}}$ should take the difference between $K_{\mathrm{VPL}}$ in (4) and $K_{\mathrm{HMI}}$ in (7) into account.

First, in order to settle the first issue, we establish an empirical method for computing $\boldsymbol{P}_{\mathrm{SCE}}$ as

$$
\boldsymbol{P}_{\mathrm{SCE}}=\frac{s^{2}}{\min _{\text {user } \in \text { service area }}}\left\{\boldsymbol{I}^{\mathrm{T}} \boldsymbol{P}_{\text {esti }} \boldsymbol{I}\right\} \boldsymbol{P}_{\text {esti }}
$$

where $\boldsymbol{I}$ is the same $\boldsymbol{I}$ in (2) and $\min _{\text {user } \in \text { service area }}\left\{\boldsymbol{I}^{\mathrm{T}} \boldsymbol{P}_{\text {esti }} \boldsymbol{I}\right\}$ is the minimum projection of $\boldsymbol{P}_{\text {esti }}$ in the SBAS service area. The most important part is how to compute $s . \sigma_{\mathrm{SCE}}^{2}$ computed from $\boldsymbol{P}_{\mathrm{SCE}}$ given by (2) must over bound the URE after the application of SCE corrections. Because of the cancellation of fast correction in the DFMC SBAS, there may be a non-zero mean of the URE, which must be taken into consideration when computing $s$.

URE is always thought to be normally distributed. Considering that $\operatorname{Pr}\left[\left|x-\mu_{x}\right|<K_{\mathrm{HMI}} \sigma_{x}\right]<1-P_{\mathrm{HMI}}$ holds for any $x \sim N\left(\mu_{x}, \sigma_{x}^{2}\right)$, and that $|x|-\left|\mu_{x}\right| \leqslant\left|x-\mu_{x}\right|$, we have

$$
\operatorname{Pr}\left[|x|<K_{\mathrm{HMI}}\left(\frac{\left|\mu_{x}\right|}{K_{\mathrm{HMI}}}+\sigma_{x}\right)\right]<1-P_{\mathrm{HMI}} .
$$

Thus, the computation of $s$ is given by

$$
s=|\operatorname{mean}(\{\mathrm{URE}\})| / K_{\mathrm{HMI}}+\operatorname{std}(\{\mathrm{URE}\})
$$

where $\{$ URE $\}$ is the dataset of URE after applying SCE corrections within $6 \mathrm{~s}$, and mean( ) and $\operatorname{std}($ ) compute the mean value and the standard deviation, respectively. We choose URE within 6 s because the alert time of a precision approach is $6 \mathrm{~s}$, and the time-varying component of URE can be reflected.

Equation (22) cannot be used because URE is unknown in real time. The only data we can use are residual range errors, which are the same as the elements of $Y$ in (3). Therefore, we rewrite (22) as

$$
s_{1}=|\operatorname{mean}(\{\Delta \rho\})| / K_{\mathrm{HMI}}+\operatorname{std}(\{\Delta \rho\})
$$


where $\{\Delta \rho\}$ is the dataset of residual range errors after applying SCE corrections within $6 \mathrm{~s}$. The advantage of (23) is that threats resulting in small range error are protected against, while threats resulting in large range error have been detected and isolated in measurement processing. Our method to isolate a large range error among measurements of a satellite is that if the difference between the residual range error, which has the largest difference from the mean of all residual range errors, and the mean of the rest residual range errors is larger than 3.29 times the standard deviation of the rest residual range errors, the residual range error is isolated. Thus, the second issue is settled by (23).

The problem with this adjustment is that $\Delta \rho$ can be close to zero when a URE is opposite to the other components of residual range error, which makes $s_{1}$ close to zero and may lead to an integrity failure. Thus, a further adjustment is made:

$$
s=\left\{\begin{array}{l}
s_{1}, \quad s_{1} \geqslant s_{\min } \\
s_{\min }, \quad \text { else }
\end{array}\right.
$$

where $s_{\min }$ is computed using (22) and historical data.

Next, the difference between $\boldsymbol{X}_{F, b r d c}$ and $\boldsymbol{X}_{\text {est } i}$ and the difference between $K_{\mathrm{VPL}}$ and $K_{\mathrm{HMI}}$ are taken into account using the algorithm proposed by Blanch et al. [10] as

$$
\begin{gathered}
\boldsymbol{P}_{S \mathrm{CE}}=\frac{s^{2}}{\min _{\text {user } \in \text { service area }}\left\{\boldsymbol{I}^{\mathrm{T}} \boldsymbol{P}_{\text {esti }} \boldsymbol{I}\right\}} . \\
\left(\frac{K_{\mathrm{HMI}}+K_{b r d c}}{K_{\mathrm{VPL}}}\right)^{2} \boldsymbol{P}_{\text {esti }}
\end{gathered}
$$

where $K_{b r d c}$ is the upper bound of the difference between $\boldsymbol{X}_{F, b r d c}$ and $\boldsymbol{X}_{e s t i}$ in range domain, and is given by

$$
K_{b r d c}=\sqrt{\left(\boldsymbol{X}_{F, b r d c}-\boldsymbol{X}_{e s t i}\right)^{\mathrm{T}} \boldsymbol{P}_{e s t i}^{-1}\left(\boldsymbol{X}_{F, b r d c}-\boldsymbol{X}_{e s t i}\right)} .
$$

\subsection{Computation of covariance matrix parameters}

Covariance matrix parameters in DFMC SBAS messages include scale exponent, Cholesky factorization elements and dual-frequency range error indicator (DFREI). If no old but active parameter is used, DFMC SBAS users compute $\sigma_{\text {SCE_DF }}^{2}$ as follows:

$$
\sigma_{\mathrm{SCE} \_\mathrm{DF}}^{2}=\left(\sigma_{\mathrm{DFRE}} \cdot \delta_{\mathrm{DFRE}}\right)^{2}
$$

where the correspondence between DFREI and $\sigma_{\mathrm{DFRE}}$ is broadcast in DFMC SBAS Message Type $37 . \delta_{\mathrm{DFRE}}$ is computed as follows:

$$
\delta_{\mathrm{DFRE}}=\sqrt{\boldsymbol{I}^{\mathrm{T}} \boldsymbol{C I}}+\varepsilon_{C}
$$

where $\boldsymbol{I}$ is the same $\boldsymbol{I}$ in (2); $\boldsymbol{C}$ is computed as follows:

$$
\left\{\begin{array}{l}
\boldsymbol{C}=\boldsymbol{R}^{\mathrm{T}} \cdot \boldsymbol{R} \\
\boldsymbol{R}=2^{\text {scale exponent }-5} \cdot\left[\begin{array}{cccc}
E_{1,1} & E_{1,2} & E_{1,3} & E_{1,4} \\
0 & E_{2,2} & E_{2,3} & E_{2,4} \\
0 & 0 & E_{3,3} & E_{3,4} \\
0 & 0 & 0 & E_{4,4}
\end{array}\right]
\end{array}\right.
$$

with $E_{i, j}$ being the Cholesky factorization element; $\varepsilon_{C}$ is to compensate for the error introduced by quantization. If the quantization error is taken into account when computing covariance matrix parameters, $\varepsilon_{C}$ is zero, and (27) can be rewritten as

$$
\left\{\begin{array}{l}
\sigma_{\mathrm{SCE} \_\mathrm{DF}}^{2}=\boldsymbol{I}^{\mathrm{T}} \boldsymbol{P}_{\mathrm{SCE}, b r d c} \boldsymbol{I} \\
\boldsymbol{P}_{\mathrm{SCE}, b r d c}=\sigma_{\mathrm{DFRE}}^{2} \boldsymbol{C}
\end{array} .\right.
$$

The scale exponent and Cholesky factorization elements are broadcast together with SCE corrections in Message Type 32, while DFREI is broadcast in Message Type 32 and Message Type 34-36. If Message Type 32 needs to be updated, the pseudo code to compute covariance matrix parameters is given as follows:

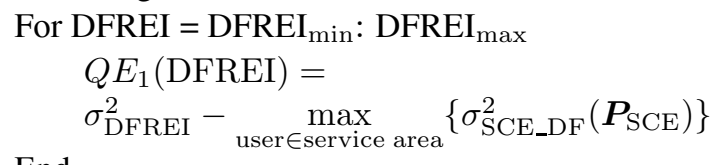

Find the DFREI whose $Q E_{1}$ is positive and minimum

$$
\begin{aligned}
& \boldsymbol{C}=\boldsymbol{P}_{\mathrm{SCE}} / \sigma_{\mathrm{DFREI}}^{2} \\
& \boldsymbol{R}=\operatorname{chol}(\boldsymbol{C}) \\
& \text { For scale exponent }=0: 7 \\
& \quad \boldsymbol{E}=\operatorname{round}\left(\boldsymbol{R} / 2^{\text {scale exponent-5 }}\right) \\
& \quad \boldsymbol{R}_{Q}=2^{\text {scale exponent-5} \times \boldsymbol{E}} \\
& \quad \boldsymbol{C}_{Q}=\boldsymbol{R}_{Q}^{\mathrm{T}} \boldsymbol{R}_{Q} \\
& \quad \boldsymbol{P}_{\mathrm{SCE}, b r d c}=\sigma_{\mathrm{DFREI}}^{2} \times \boldsymbol{C}_{Q} \\
& \quad Q E_{2}(\text { scale exponent, } \boldsymbol{E})= \\
& \quad \max _{\text {user } \in \text { service area }}\left\{\sigma_{\mathrm{SCE} \_D F}^{2}\left(\boldsymbol{P}_{\mathrm{SCE}, b r d c}-\boldsymbol{P}_{\mathrm{SCE}}\right)\right\}
\end{aligned}
$$$$
\text { End }
$$

Find the combination of scale exponent and Cholesky factorization elements whose $Q E_{2}$ is positive and minimum. Thus, the broadcast values of DFREI, scale exponent and Cholesky factorization elements are determined.

DFREI $_{\min }$ and $\mathrm{DFREI}_{\max }$ are the minimum and the maximum of the range of DFREI, which is determined by DFMC SBAS provider in advance; $\operatorname{chol}()$ is the Cholesky factorization function; $\operatorname{round}(x)$ rounds $x$ to the nearest integer; $\max _{\text {user } \in \text { service area }}\left\{\sigma_{\mathrm{SCE} \_\mathrm{DF}}^{2}\left(\boldsymbol{P}_{\mathrm{SCE}}\right)\right\}$ is the maximum projection of $\boldsymbol{P}_{\mathrm{SCE}}$ within the service area; $\underset{\text { user } \in \text { service area }}{\max }\left\{\sigma_{\mathrm{SCE} \_D F}^{2}\left(\boldsymbol{P}_{\mathrm{SCE}, b r d c}-\boldsymbol{P}_{\mathrm{SCE}}\right)\right\}$ is the maximum difference between the projection of $\boldsymbol{P}_{\mathrm{SCE}, b r d c}$ and that of $\boldsymbol{P}_{\mathrm{SCE}}$ within the service area. The reason that the 
chosen $Q E_{1}$ and $Q E_{2}$ must be positive is that the projection of $\boldsymbol{P}_{\mathrm{SCE}, b r d c}$ must be larger than that of $\boldsymbol{P}_{\mathrm{SCE}}$, so that the quantization error is included in $\boldsymbol{P}_{\mathrm{SCE}, b r d c}$.

If Message Type 34-36 have not been updated for more than $6 \mathrm{~s}$, or the DFERI in previous message cannot bound URE, DFREI needs to be updated. Thus, $\boldsymbol{P}_{\mathrm{SCE}}$ is computed every second. The pseudo code to compute DFREI is given as follows:

$\boldsymbol{R}_{Q}=2^{\text {scale exponent-5 }} \times \boldsymbol{E}$ with scale exponent and $\boldsymbol{E}$ broadcast in the active Message Type 32

$$
\begin{aligned}
& \boldsymbol{C}_{Q}=\boldsymbol{R}_{Q}^{\mathrm{T}} \boldsymbol{R}_{Q} \\
& \text { For DFREI }=\mathrm{DFREI}_{\min }: \mathrm{DFREI}_{\max } \\
& \quad \boldsymbol{P}_{\mathrm{SCE}, b r d c}=\sigma_{\mathrm{DFREI}}^{2} \times \boldsymbol{C}_{Q} \\
& \quad \underset{Q E(\mathrm{DFREI})=}{\quad \max _{\text {user } \in \text { service area }}\left\{\sigma_{\mathrm{SCE} \_\mathrm{DF}}^{2}\left(\boldsymbol{P}_{\mathrm{SCE}, b r d c}-\boldsymbol{P}_{\mathrm{SCE}}\right)\right\}}
\end{aligned}
$$$$
\text { End }
$$

Find the DFREI whose $Q E$ is positive and minimum.

\section{Performance evaluation}

The data used in the performance evaluation include the GPS broadcast message and the GPS precise ephemeris provided by the National Geospatial-intelligence Agency (NGA), the WAAS broadcast message and WAAS stations' antenna L1 phase center positions provided by the National Satellite Test Bed, and measurements made by WAAS reference stations and provided by the National Geodetic Survey (NGS). Please note that WAAS has 38 stations and each station has three independent receivers. However, NGS provides 37 stations' measurements made at $1 \mathrm{~Hz}$, and measurements of GPS L1 C/A signal and L2 $\mathrm{P}(\mathrm{Y})$ signal made by only one receiver of each station are available. Therofore, measurements of GPS L2 P(2) signals are used instead of GPS L5 signals. Parameters, including SCE correction, scale exponent, Cholesky factorization elements and DFREI, in Message Type 32 are updated every $120 \mathrm{~s}$. DFREI in Message Type 35 is updated if Message Type 35 have not been updated for more than $6 \mathrm{~s}$, or the DFERI in previous message cannot bound current URE. The correspondence between DFREI and $\sigma_{\text {DFRE }}$ is listed in Table 1.

Table 1 Correspondence between DFREI and $\sigma_{\text {DFRE }}$ m

\begin{tabular}{cc||cc}
\hline DFREI & $\sigma_{\text {DFRE }}$ & DFREI & $\sigma_{\text {DFRE }}$ \\
\hline 0 & 0.125 & 8 & 1.5 \\
1 & 0.25 & 9 & 1.75 \\
2 & 0.375 & 10 & 2 \\
3 & 0.5 & 11 & 2.5 \\
4 & 0.625 & 12 & 3 \\
5 & 0.75 & 13 & 4 \\
6 & 1 & 14 & 10 \\
7 & 1.25 & & \\
\hline
\end{tabular}

DFREI $_{\text {min }}$ and DFREI ${ }_{\text {max }}$ are 0 and 14 . The results presented below are obtained from data from January 1 to December 31, 2016. The sampling interval is $6 \mathrm{~s}$. Results of
GPS PRN 4 are not available, because it is not assigned to a healthy GPS satellite.

\subsection{SIS accuracy}

The SCE correction error is defined as

$$
\left[\begin{array}{c}
\Delta x \\
\Delta y \\
\Delta z \\
\Delta t
\end{array}\right]=\left[\begin{array}{l}
x \\
y \\
z \\
t
\end{array}\right]_{\mathrm{SBAS}}-\left[\begin{array}{l}
x \\
y \\
z \\
t
\end{array}\right]_{P E}
$$

where $\left[\begin{array}{llll}x & y & z & t\end{array}\right]_{\mathrm{SBAS}}^{\mathrm{T}}$ is given by (1), and $\left[\begin{array}{llll}x & y & z & t\end{array}\right]_{P E}^{\mathrm{T}} \mathrm{de}-$ notes the SCE values interpolated with the NGA precise ephemeris employing the Lagrange interpolation, which are treated as the SCE true values. Fig. 2 shows that, for most satellites, RMS values of SCE correction errors obtained using SCE corrections computed by the AK method are markedly smaller than that using SCE corrections broadcast by WAAS. As listed in Table 2, the RMS values of the ephemeris correction error of the GPS constellation using SCE corrections computed by the AK method are reduced by $79.2 \%, 79.2 \%$ and $83.3 \%$, respectively, and the RMS value of the clock correction error of GPS constellation is reduced by $67.5 \%$.

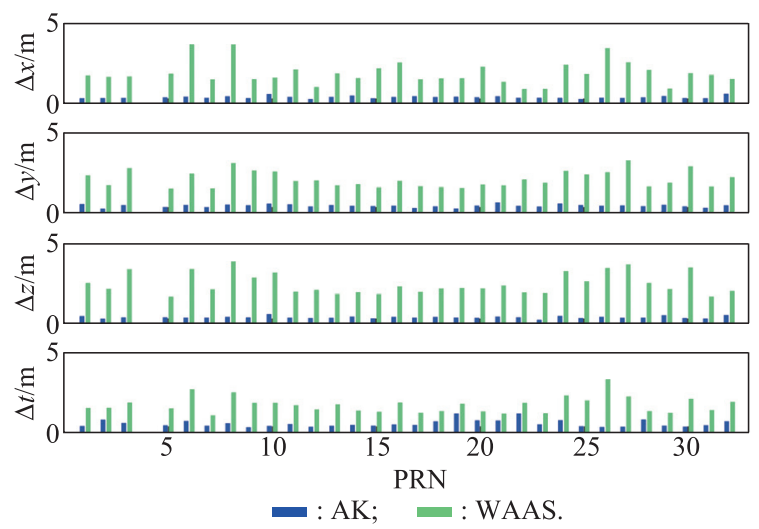

Fig. 2 RMS values of SCE correction error for each GPS satellite

Table 2 RMS values of SCE correction error for GPS constellation

\begin{tabular}{ccc}
\hline Correction error & AK & WAAS \\
\hline$\Delta x$ & 0.436 & 2.099 \\
$\Delta y$ & 0.451 & 2.171 \\
$\Delta z$ & 0.434 & 2.598 \\
$\Delta t$ & 0.580 & 1.786 \\
\hline
\end{tabular}

SCE correction error affects the position accuracy in the way of URE. URE is thus an indicator of the SCE correction accuracy in the range domain. There are three UREs involved here: $\mathrm{URE}_{\mathrm{AK}}$ refers to the URE after applying SCE corrections computed by the AK method, URE $E_{L T}$ refers to the URE after applying SCE corrections broadcast by the WAAS and URE $\mathrm{FLT}_{\mathrm{F}}$ refers to the URE after applying SCE corrections and fast corrections broadcast by 
the WAAS. The statistics of URE of the GPS constellation is given in Table 3. Taking station BET1 as an example, $\mathrm{URE}_{\mathrm{AK}}$ is reduced by $46.1 \%, 40.8 \%$ and $79.7 \%$ with respect to $\mathrm{URE}_{\mathrm{LT}}$, and $15.8 \%, 17.5 \%$ and $11.9 \%$ with re-

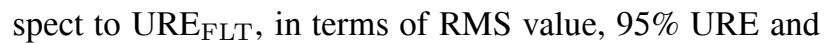
maximum URE, respectively. The results also confirm that fast correction plays an important role in improving range accuracy.

Table 3 Statistics of URE of GPS constellation

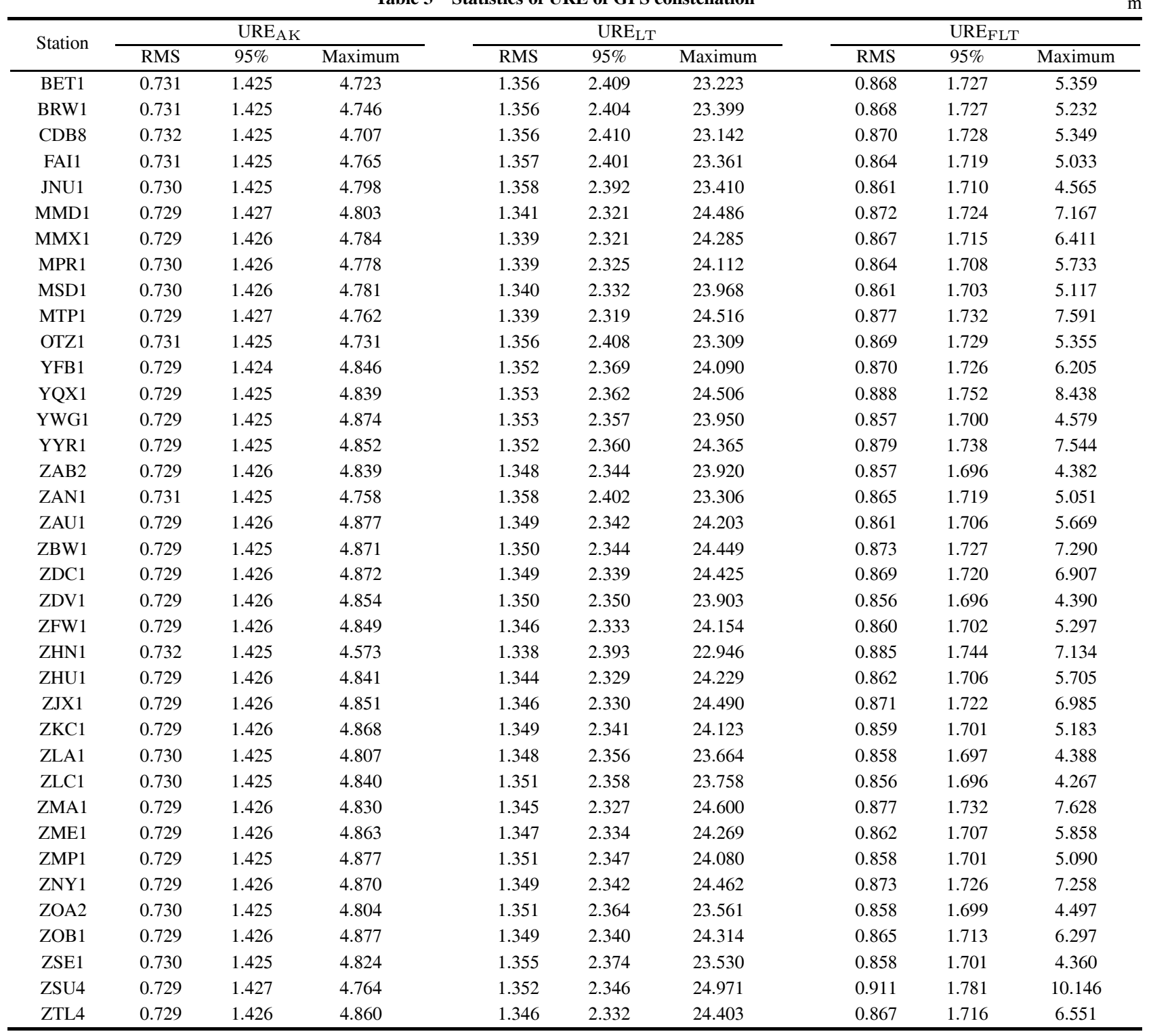

\subsection{SIS integrity}

The normalized URE (NURE) is used to simplify the description of SIS integrity, which is defined as

$$
\mathrm{NURE}=\frac{\mathrm{URE}}{\sigma_{\mathrm{SCE} \_D F}} .
$$

Considering that $P_{\mathrm{HMI}}$ is allocated to be $1 \times 10^{-7} / 150 \mathrm{~s}$ in [22], DFREI is updated every $6 \mathrm{~s}$ when SIS is fault-free, and the sampling interval here is $6 \mathrm{~s}, P_{\mathrm{HMI}}$ in (7) is set to be $4 \times 10^{-9} / 6 \mathrm{~s}$. Thus, the SIS integrity requirement is given by

$$
\operatorname{Pr}[|\mathrm{NURE}|<5.8842]<1-4 \times 10^{-9} .
$$

The NURE dataset of the WAAS is computed using URE $_{\text {FLT }}$ because the integrity parameters broadcast by the WAAS describe the model variance of URE $E_{F L T}$, while the NURE dataset of the AK method is computed by using $\mathrm{URE}_{\mathrm{AK}}$ and $\sigma_{\mathrm{SCE}} \mathrm{DF}$ computed by using covariance matrix parameters. There are 3046349823 samples and 3046304054 samples in WAAS dataset and AK dataset, respectively. The probability distribution curves of the 
WAAS dataset, the AK dataset and a dataset of one million samples obtained by sampling the standard normal distribution are plotted in Fig. 3. The maximum absolute values of WAAS dataset and AK dataset are 4.198 and 3.810. There is no sample from the AK dataset whose absolute value is larger than 5.8842 , indicating that AK dataset meets the SIS integrity requirement described in (33).

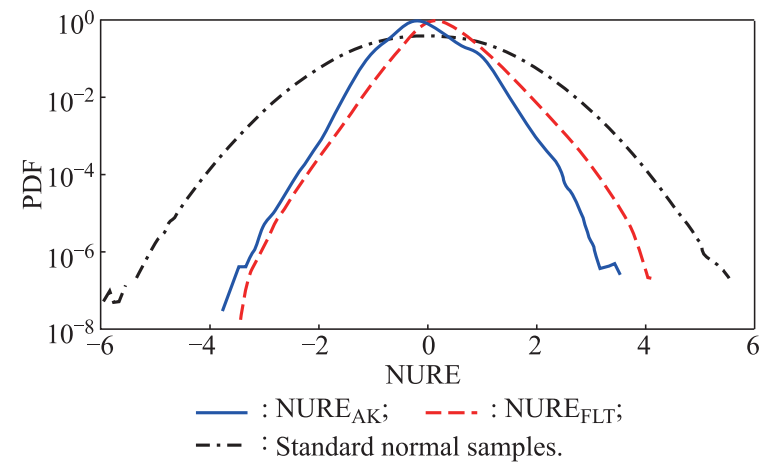

Fig. 3 Probability distribution curves

The GPS constellation was in normal operations from January 1 to November 30, 2016. Two simulation cases are designed to analyze the integrity further. The first case is to find out what will happen when a clock anomaly occurs, and the second case is to find out what will happen when an error exists in SCE clock prediction. Considering the SIS accuracy standards of the GPS standard position service [23], two types of clock anomaly are set in each case. In the first case, a 6-meter step anomaly was added to the GPS PRN 1 satellite clock from $1900 \mathrm{~s}$ to $2049 \mathrm{~s}$ on January 1, 2016, and a slope anomaly with the rate of $0.06 \mathrm{~m} / \mathrm{s}$ was added to the GPS PRN 1 satellite clock from 2200 s to 2349 s on January 1, 2016. In the second case, a $6 \mathrm{~m}$ step error was added to the GPS PRN 1 satellite clock prediction from $1900 \mathrm{~s}$ to $2049 \mathrm{~s}$ on January 1, 2016, and a slope error with the rate of $0.06 \mathrm{~m} / \mathrm{s}$ was added to the GPS PRN 1 satellite clock prediction from $2200 \mathrm{~s}$ to $2349 \mathrm{~s}$ on January 1, 2016, while covariance of $X_{a p}$ is kept unchanged. GPS PRN 1 satellite's elevation angle is around $10^{\circ}$ during the period. Station BET1 is selected to be the user. In these two cases, the sampling interval is changed to $1 \mathrm{~s}$. Same as the result with no manually added fault as shown in Fig. 4, there is no integrity risk in both cases as shown in Fig. 5 and Fig. 6.

In Fig. 5, when the clock anomaly happens, SCE corrections computed by the $\mathrm{AK}$ method cannot correct the anomaly, and when the clock anomaly ends, URE is still at the serval-meter level. The main reason is that SCE error estimations within the most recent $900 \mathrm{~s}$ are used to compute SCE corrections. After the clock anomaly happens at $1900 \mathrm{~s}, \sigma_{\mathrm{SCE} \_} D F$ is significantly larger than that in Fig. 4 , especially around $1900 \mathrm{~s}, 2050 \mathrm{~s}$ and $2350 \mathrm{~s}$. The main reason is that both normal $\Delta \rho$ and abnormal $\Delta \rho$ are used in (24) at those epochs, increasing the standard deviation of $\{\Delta \rho\}$. URE is bounded all the time, SIS integrity is thus ensured when the clock anomalies happen in the first case.

In Fig. 6, URE is slightly larger than that in Fig. 4, indicating that clock prediction error has been corrected by real-time measurements.

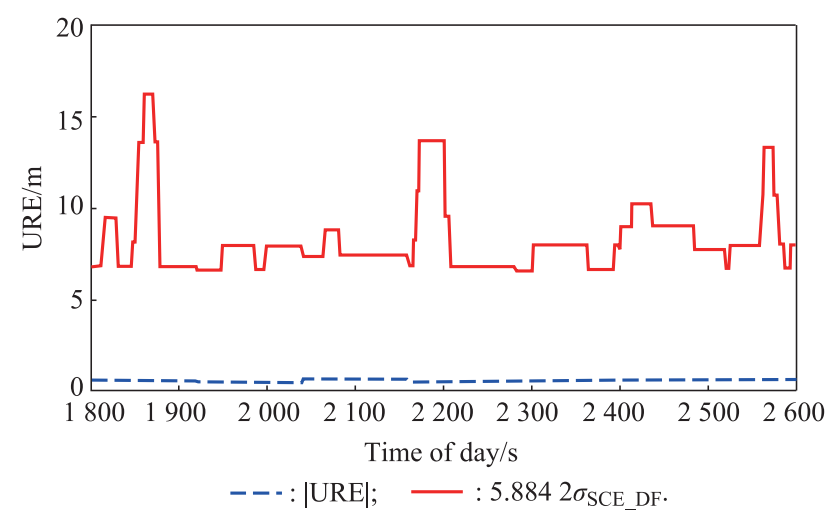

Fig. 4 No clock anomaly or prediction error

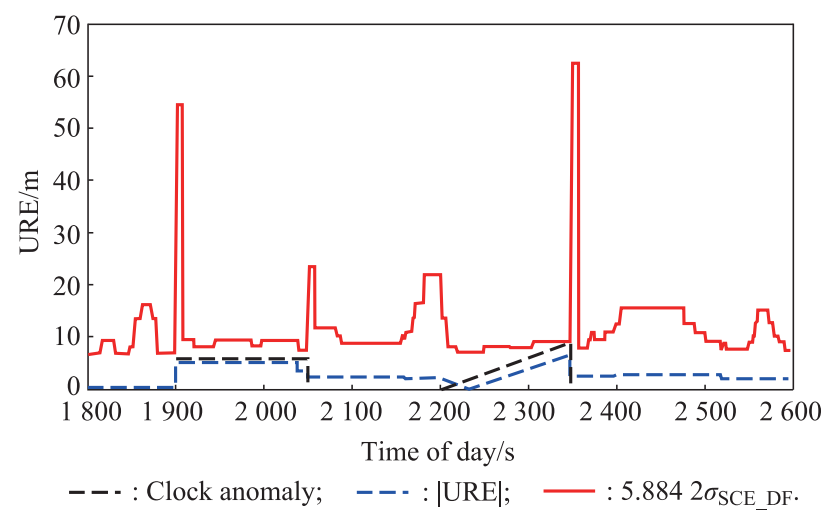

Fig. 5 Adding clock anomaly

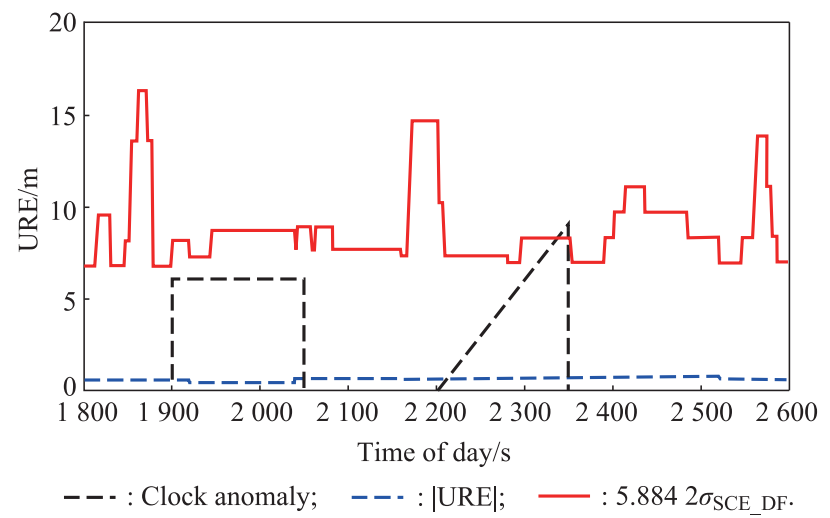

Fig. 6 Adding clock prediction error

After the clock prediction error happens at $1900 \mathrm{~s}$, $\sigma_{\text {SCE_DF }}$ changes little compared with that in Fig. 4. URE is bounded all the time, SIS integrity is thus ensured when the clock errors exist in the SCE predictions in the second case. 


\subsection{Position performance}

The performance of SCE augmentation parameters is finally evaluated in the position domain. Please note that minimum operational performance specification (MOPS) for DFMC SBAS is under developing. The position algorithm used here is based on the MOPS for single-frequency SBAS. One of the modifications is that the iono-free smoother [24] is used, thus the ionosphere related items are removed. $\sigma_{\text {tropo }}^{2}$ in (6) is the same as that in singlefrequency SBAS. Because of the combination of L1 and L2, $\sigma_{\text {air_DF }}^{2}$ in (6) is computed as follows:

$\sigma_{\text {air_DF }}^{2}=\left(\left(\frac{f_{L 1}^{2}}{f_{L 1}^{2}-f_{L 2}^{2}}\right)^{2}+\left(\frac{f_{L 2}^{2}}{f_{L 1}^{2}-f_{L 2}^{2}}\right)^{2}\right) \sigma_{\text {air_SF }}^{2}$

where $f_{L 1}$ and $f_{L 2}$ are the center frequencies of GPS L1 and L2, and the computation method of $\sigma_{\text {air_DF }}^{2}$ is given in RTCA DO-229. AK navigation solutions use SCE correction and integrity information computed by the AK method. WAAS navigation solutions use SCE correction, fast correction and integrity information broadcast by WAAS.

The protection levels with the augmentation parameters computed by using the AK method and those with WAAS augmentation parameters are plotted in Fig. 7. The maximum values of $99 \%$ HPL and 99\% VPL of AK navigation solutions among all stations are $20.970 \mathrm{~m}$ and $27.257 \mathrm{~m}$, respectively, while those of WAAS navigation solutions are $39.503 \mathrm{~m}$ and $50.507 \mathrm{~m}$, respectively.

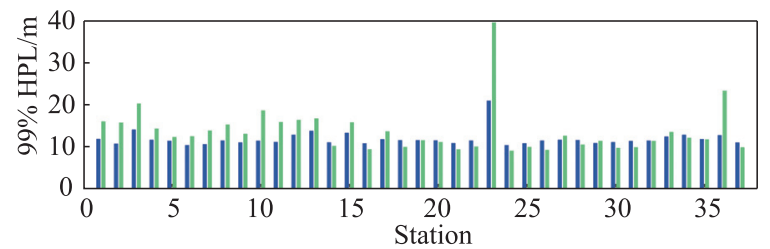

(a) $99 \% \mathrm{HPL}$

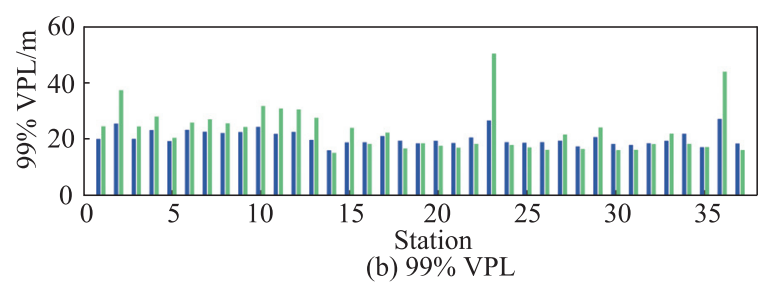

- : AK; $=$ : WAAS.

Fig. 7 Protection level

The service availability is defined as the portion of time that VPL is smaller than VAL. The availabilities of CAT-I (VAL of $15 \mathrm{~m}$ ) of AK navigation solutions and WAAS navigation solutions are listed in Table 4 , and are plotted in Fig. 8 and Fig. 9, respectively. With the GPS constellation only, the CAT-I (VAL $=15 \mathrm{~m}$ ) availability of AK navigation solutions in the continental United States is about $90 \%$, while that of WAAS navigation solutions is about $95 \%$.

Table 4 Availabilities of CAT-I (VAL=15 m)

\begin{tabular}{ccc||ccc}
\multicolumn{7}{c}{ Table 4 } & \multicolumn{1}{c}{ Availabilities of CAT-I (VAL=15 m) } \\
\hline Station & AK & WAAS & Station & AK & WAAS \\
\cline { 5 - 6 } BET1 & 85.5 & 44.5 & ZDC1 & 90.7 & 94.3 \\
BRW1 & 58.6 & 3.5 & ZDV1 & 93.2 & 97.5 \\
CDB8 & 85.3 & 43.5 & ZFW1 & 89.8 & 97.2 \\
FAI1 & 82.6 & 51.6 & ZHN1 & 51.8 & 0.0 \\
JNU1 & 84.9 & 72.7 & ZHU1 & 89.6 & 95.3 \\
MMD1 & 81.6 & 63.2 & ZJX1 & 85.7 & 90.5 \\
MMX1 & 79.7 & 42.9 & ZKC1 & 91.2 & 98.0 \\
MPR1 & 81.5 & 41.3 & ZLA1 & 88.0 & 79.4 \\
MSD1 & 82.2 & 45.7 & ZLC1 & 93.2 & 96.9 \\
MTP1 & 72.4 & 12.9 & ZMA1 & 81.1 & 70.8 \\
OTZ1 & 76.9 & 15.5 & ZME1 & 90.8 & 97.7 \\
YFB1 & 84.7 & 15.3 & ZMP1 & 94.2 & 97.7 \\
YQX1 & 86.4 & 16.9 & ZNY1 & 92.8 & 92.7 \\
YWG1 & 96.9 & 98.6 & ZOA2 & 87.8 & 76.3 \\
YYR1 & 91.2 & 36.8 & ZOB1 & 85.0 & 92.0 \\
ZAB2 & 92.0 & 97.1 & ZSE1 & 95.0 & 94.0 \\
ZAN1 & 87.4 & 67.2 & ZSU4 & 66.1 & 0.2 \\
ZAU1 & 90.9 & 96.5 & ZTL4 & 88.8 & 95.2 \\
ZBW1 & 93.9 & 90.9 & & & \\
\hline
\end{tabular}

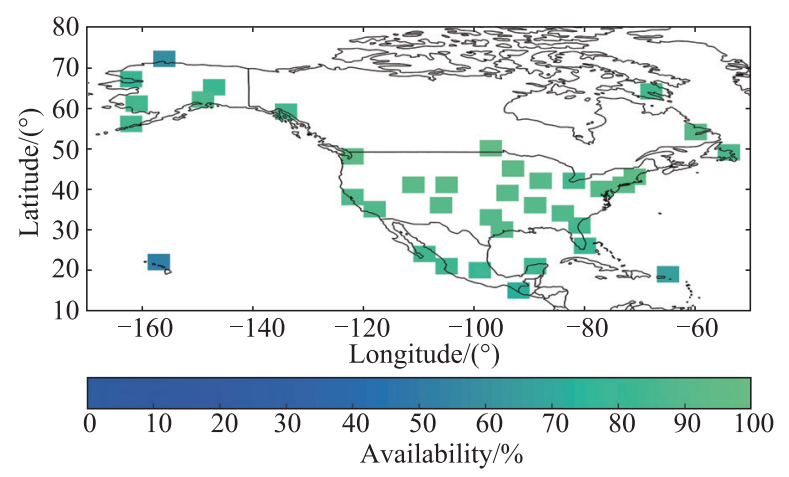

Fig. 8 CAT-I $(\mathrm{VAL}=15 \mathrm{~m})$ availability of AK navigation solutions

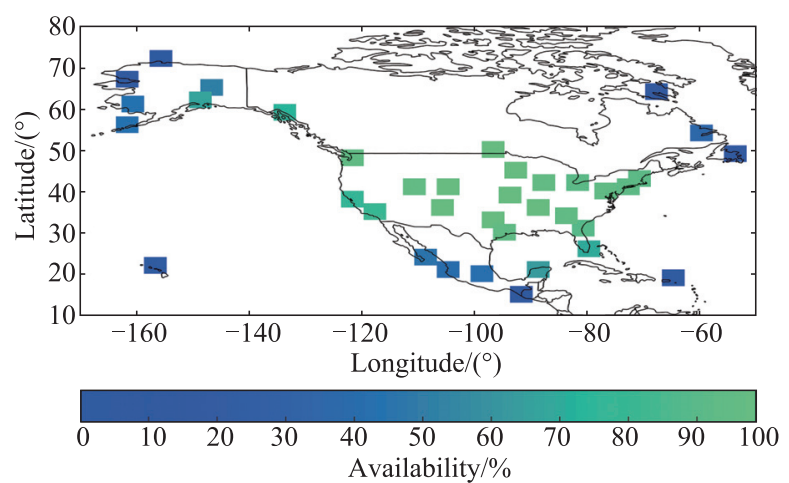

Fig. 9 CAT-I (VAL=15 m) availability of WAAS navigation solutions

Meanwhile, the CAT-I (VAL $=15 \mathrm{~m}$ ) availability of AK navigation solutions in Alaska and other area is markedly larger than that of WAAS navigation solutions, indicating that the shortcoming mentioned in the problem statement section has been overcome by the AK method. Thus, the 
AK method is of value to improve the service performance near the boundary of the service area.

\section{Preliminary results with CMONOC data}

The AK method is applied to process measurements collected by CMONOC stations to find out the potential performance in China. Please note that receivers used in CMONOC stations are Trimble NetR8/R9 receivers and are not capable of being SBAS monitoring receivers. CMONOC provides GPS L1 C/A and L2 P(Y) measurements and GLONASS L1 and L2 measurements made at $1 \mathrm{~Hz}$. There are 35 CMONOC stations chosen to be simulated SBAS stations. The results presented below are obtained from data from July 24 to July 31, 2016.

Table 5 gives 99\% HPL and 99\% VPL of each station's navigation solutions using GPS constellation only.

\begin{tabular}{|c|c|c|}
\hline Station & $99 \%$ HPL & $99 \%$ VPL \\
\hline FJXP & 83.618 & 234.891 \\
\hline GDZH & 105.599 & 285.665 \\
\hline GDZJ & 102.431 & 225.053 \\
\hline GSDH & 69.261 & 81.385 \\
\hline GXHC & 69.188 & 172.926 \\
\hline HBES & 41.825 & 134.463 \\
\hline HLMH & 38.670 & 52.066 \\
\hline HLWD & 34.733 & 56.863 \\
\hline HNLY & 60.305 & 160.630 \\
\hline JLCB & 38.018 & 76.086 \\
\hline JLCL & 34.763 & 57.031 \\
\hline JSLS & 47.076 & 150.357 \\
\hline NMDW & 35.713 & 61.403 \\
\hline NMEJ & 42.521 & 83.628 \\
\hline NMEL & 34.099 & 103.720 \\
\hline NMWT & 33.203 & 76.501 \\
\hline QHGC & 28.736 & 84.658 \\
\hline SCLH & 25.559 & 90.178 \\
\hline SCYY & 55.825 & 115.929 \\
\hline SDJX & 29.268 & 142.944 \\
\hline SNMX & 37.568 & 141.162 \\
\hline TJBD & 28.835 & 150.096 \\
\hline XJAL & 64.122 & 74.082 \\
\hline XJBC & 66.967 & 71.303 \\
\hline XJDS & 55.104 & 68.001 \\
\hline XJHT & 72.067 & 91.013 \\
\hline XJML & 115.335 & 119.324 \\
\hline XJRQ & 55.731 & 75.732 \\
\hline XJZS & 62.311 & 68.073 \\
\hline XZBG & 55.162 & 157.675 \\
\hline XZNM & 55.405 & 154.254 \\
\hline XZRK & 59.612 & 202.861 \\
\hline YANC & 28.575 & 91.510 \\
\hline YNRL & 152.828 & 335.959 \\
\hline YNWS & 69.421 & 225.789 \\
\hline
\end{tabular}

The reason for protection level using CMONOC measurements being larger than that using WAAS measurements is that the CMONOC receiver has fewer satellites in view. The latitudes of station ZAB2 from WAAS network and station SDJX from CMONOC network are both around $35^{\circ}$. As shown in Fig. 10, the average of satellites in view of ZAB2 is about 10, while that of SDJX is about 5.5. A few satellites are excluded because of low signalnoise ratio. With fewer satellites in view, it is reasonable that HPL and VPL are larger.

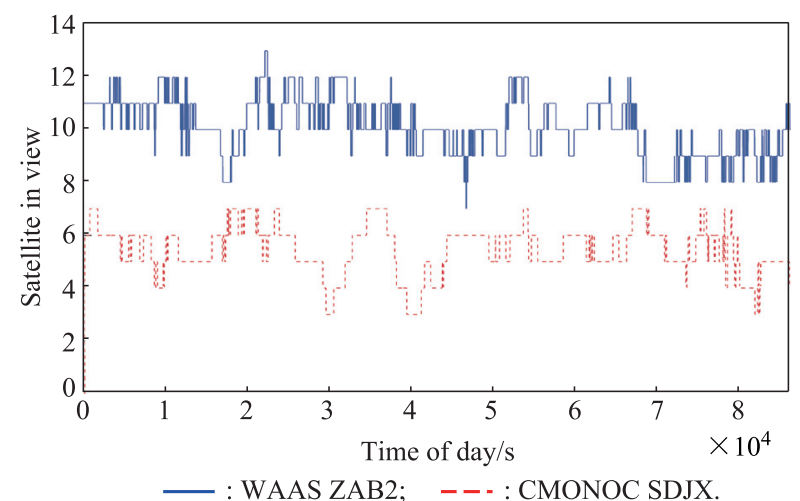

Fig. 10 Satellites in view

Table 6 gives those using GPS and GLONASS constellations.

Table 6 Protection level with GPS and GLONASS contellations

\begin{tabular}{ccc}
\hline Station & $99 \%$ HPL & $99 \%$ VPL \\
\hline FJXP & 7.462 & 18.351 \\
GDZH & 9.279 & 25.889 \\
GDZJ & 8.643 & 29.453 \\
GSDH & 6.724 & 14.986 \\
GXHC & 7.718 & 28.131 \\
HBES & 7.677 & 24.899 \\
HLMH & 6.528 & 11.003 \\
HLWD & 7.506 & 15.560 \\
HNLY & 7.059 & 21.217 \\
JLCB & 6.973 & 18.602 \\
JLCL & 6.428 & 11.609 \\
JSLS & 7.339 & 21.237 \\
NMDW & 6.107 & 11.390 \\
NMEJ & 6.470 & 13.294 \\
NMEL & 6.707 & 11.345 \\
NMWT & 6.116 & 11.672 \\
QHGC & 9.822 & 26.271 \\
SCLH & 7.497 & 18.956 \\
SCYY & 9.608 & 21.913 \\
SDJX & 6.706 & 12.712 \\
SNMX & 7.547 & 21.225 \\
TJBD & 6.270 & 11.999 \\
XJAL & 6.855 & 12.814 \\
XJBC & 7.502 & 17.205 \\
XJDS & 6.903 & 14.872 \\
XJHT & 7.647 & 16.365 \\
XJML & 7.051 & 14.777 \\
XJRQ & 8.050 & 21.767 \\
XJZS & 7.046 .1762 \\
XZBG & 8.706 & 15.840 \\
XZNM & 8.379 & 23.283 \\
XZRK & 7.437 & 24.725 \\
YANC & 6.044 & 19.167 \\
YNRL & & \\
YNWS & 692 & \\
\hline & & 13.17 \\
\hline
\end{tabular}


The results show that the introduction of GLONASS constellation decreases HPL and VPL significantly. As shown in Fig. 11, all stations' CAT-I $(\mathrm{VAL}=15 \mathrm{~m})$ availability values are larger than $90 \%$. There are 13 stations whose CAT-I (VAL $=15 \mathrm{~m}$ ) availability is larger than $99 \%$, meaning that, even using CMONOC stations' receivers, users in some part of China can obtain CAT-I (VAL = $15 \mathrm{~m})$ service.

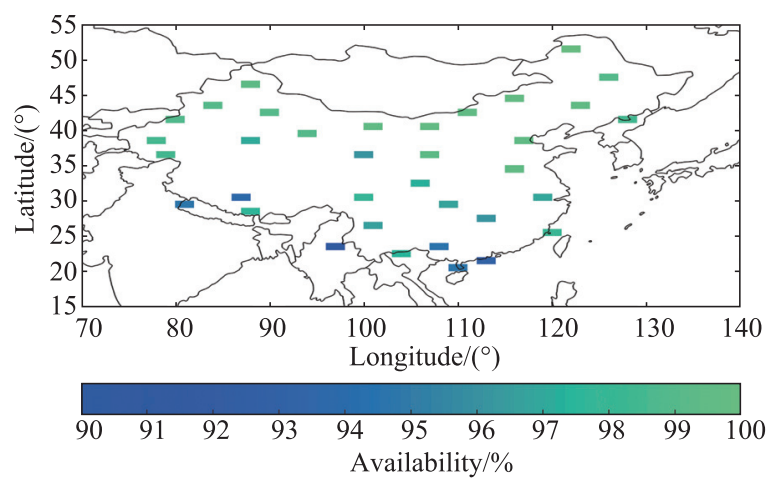

Fig. 11 CAT-I (VAL=15 m) availability of navigation solutions using GPS and GLONASS constellations

\section{Conclusions}

Based on the idea that the parameter-fitting error of GNSS broadcast message can be eliminated by introducing SCE prediction, the AK method for computing SCE corrections and covariance matrix parameters is proposed to solve the problem that a satellite at low elevation is too overbounded for DFMC SBAS. The following results are obtained in the performance evaluation of the AK method.

i) Compared with the WAAS, the SCE correction accuracy is remarkably improved. The RMS values of ephemeris correction error on each axis of the ECEF frame and clock correction error are reduced by $79.2 \%, 79.2 \%$, $83.3 \%$ and $67.5 \%$, respectively. And $\mathrm{URE}_{\mathrm{AK}}$ is reduced by $15.8 \%, 17.5 \%$ and $11.9 \%$ with respect to $\mathrm{URE}_{\mathrm{FLT}}$, in terms of RMS value, 95\% URE and maximum URE, respectively.

ii) The SIS integrity is ensured, even when a clock anomaly occurs or a clock prediction error exists.

iii) With the GPS constellation only, the CAT-I (VAL of $15 \mathrm{~m}$ ) availability of AK navigation solutions in the continental United States is about $90 \%$, while the availability in the other regions is markedly improved compared with that of WAAS navigation solutions.

The AK method is applied to process CMONOC measurements. The preliminary shows that users in some part of China can obtain CAT-I (VAL $=15 \mathrm{~m}$ ) service with GPS and GLONASS, even though the performance of a CMONOC receiver is worse than that of an SBAS monitoring receiver.

\section{Acknowledgment}

The authors would like to thank the crustal movement observation network of China (CMONOC) for providing data.

\section{References}

[1] Satellite-Based Augmentation System Interoperability Working Group. Satellite-based augmentation system dualfrequency multi-constellation definition document v2.0. 2016.

[2] ENGE P, WALTER T, PULLEN S, et al. Wide area augmentation of the global positioning system. Proceedings of the IEEE, 1996, 84(8): $1063-1088$.

[3] SHALLBERG K, SHLOSS P, ALTSHULER E, et al. WAAS measurement processing, reducing the effects of multipath. Proc. of the 14th International Technical Meeting of the Satellite Division of the Institute of Navigation, 2001: $2334-2340$.

[4] SHALLBERG K, GRABOWSKI J. Considerations for characterizing antenna induced range errors. Proc. of the 15th International Technical Meeting of the Satellite Division of the Institute of Navigation, 2002: 809-815.

[5] SHALLBERG K, SHENG F. WAAS measurement processing: current design and potential improvements. Proc. of the IEEE/ION Position, Location and Navigation Symposium, 2008: $253-262$.

[6] BLANCH J, WALTER T, ENGE P. Adapting Kriging to the WAAS MOPS ionospheric grid. Proc. of the National Technical Meeting of the Institute of Navigation, 2003: 848-853.

[7] WALTER T, DATTA-BARUA S. The impact of ionospheric storms on WAAS. Proc. of the 61st Annual Meeting of the Institute of Navigation, 2005: $222-250$.

[8] ROVIRA-GARCIA A, JUAN J M, SANZ J, et al. Accuracy of ionospheric models used in GNSS and SBAS: methodology and analysis. Journal of Geodesy, 2016, 90(3): 229-240.

[9] WU T, PECK S. An analysis of satellite integrity monitoring improvement for WAAS. Proc. of the 15th International Technical Meeting of the Satellite Division of the Institute of Navigation, 2002: 756-765.

[10] BLANCH J, WALTER T, ENGE P. A clock and ephemeris algorithm for dual frequency SBAS. Proc. of the 24th International Technical Meeting of the Satellite Division of the Institute of Navigation, 2011: 2513-2519.

[11] GREWAL M S. Space-based augmentation for global navigation satellite systems. IEEE Trans. on Ultrasonics, Ferroelectrics, and Frequency Control, 2012, 59(3): 497-503.

[12] WALTER T, BLANCH J, ENGE P. L1/L5 SBAS MOPS to support multiple constellations. Proc. of the 25th International Technical Meeting of the Satellite Division of the Institute of Navigation, 2012: 1287 - 1297.

[13] FIDALGO J, ODRIOZOLA M, CUETO M, et al. SBAS L5 enhanced ICD for aviation: definition and preliminary experimentation results. Proc. of the 27th International Technical Meeting of the Satellite Division of the Institute of Navigation, 2014: 3289-3298.

[14] FIDALGO J, ODRIOZOLA M, CUETO M, et al. SBAS L1/L5 enhanced ICD for aviation: experimentation results. Proc. of the 28th International Technical Meeting of the Satellite Division of the Institute of Navigation, 2015: 1764-1774.

[15] RTCA Special Committee 159. Minimal operational perfor- 
mance standards for global positioning system/satellite-based augmentation system airborne equipment (RTCA DO-229E). 2016.

[16] William J Hughes Technical Center. Wide area augmentation system performance analysis report \#58. http://www.nstb.tc. faa.gov/REPORTS/waaspan58.pdf.

[17] William J Hughes Technical Center. Global positioning system (GPS) standard positioning service (SPS) performance analysis report \#94. http://www.nstb.tc.faa.gov/ REPORTS/PAN95_1016.pdf.

[18] CHOI K K, RAY J, GRIFFITHS J, et al. Evaluation of GPS orbit prediction strategies for the IGS ultra-rapid products. GPS Solutions, 2013, 17(3): 403-412.

[19] GELB A. Applied optimal estimation. Cambridge: M.I.T. Press, 2001.

[20] LI X R, JILKOV V P. Survey of maneuvering target tracking, Part I: dynamic models. IEEE Trans. on Aerospace and Electronic Systems, 2003, 39(4): 1333 - 1364.

[21] SINGER R. Estimating optimal tracking filter performance for manned maneuvering targets. IEEE Trans. on Aerospace and Electronic Systems, 1970, 6(4): 473-483.

[22] ROTURIER B, CHATRE E, VENTURA-TRAVESET J. The SBAS integrity concept standardised by ICAO application to EGNOS. http://www.egnos-pro.esa.int/Publications/GNSS\%202001/SBAS_integrity.pdf.

[23] Department of Defense. GPS standard positioning service (SPS) performance standard, 4th ed. http://www.gps.gov/ technical/ps/2008-SPS-performance-standard.pdf.

[24] HWANG P Y, MCGRAW G A, BADER J R. Enhanced differential GPS carrier-smoothed code processing using dualfrequency measurements. Navigation, 1999, 46(2): 127-137.

\section{Biographies}

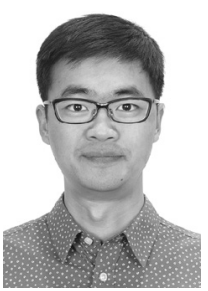

CHEN Jie was born in 1989. He received his B.S. degree from Beihang University in 2012. He is currently a Ph.D. candidate in the School of Electronic and Information Engineering at Beihang University. His research interest is satellite-based augmentation system.

E-mail: chenjiee@buaa.edu.cn

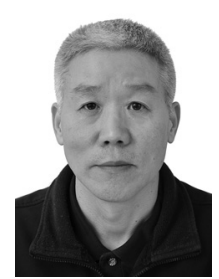

HUANG Zhigang was born in 1962. He received his Ph.D. degree from Beihang University in 2004. $\mathrm{He}$ is currently a professor in the School of Electronic and Information Engineering at Beihang University. His research interests include integrity algorithms and related software for ground-based augmentation system and satellite-based augmentation system.

E-mail: baahzg@163.com

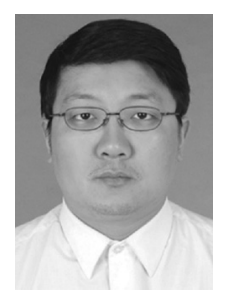

LI Rui was born in 1976. He received his Ph.D. degree from Beihang University in 2006. He is now a senior engineer in the School of Electronic and Information Engineering at Beihang University. His main interests cover required navigation performance and GNSS augmentation technologies in satellite-based augmentation system, ground-based augmentation system, and airborne based augmentation system.

E-mail: lee_ruin@263.net 